\title{
Sa Kanilang Naiibang Pag-iindak at Pamumukadkad: Performativity at Pagkalesbiyan sa mga Indie Film na Rome and Juliet at Ang Huling Cha-Cha ni Anita
}

\author{
Judith R. Angeles, \\ Tracie Kathlynne B. Bacarro, \\ F.P.A. Demeterio, III, \\ Jonathan Vergara Geronimo, and \\ Patricia Bettina L. Peliño
}

\begin{abstract}
This paper features two Filipino independent films that discuss the relatively avoided topic of lesbianism in the Philippines. The "indie" films Rome and Juliet (2006) by Connie Macatuno and Ang Huling Cha-Cha ni Anita (2013) by Sigrid Andre Bernardo are comparatively studied to present the different images of the lesbian in the Filipino experience. This study relied upon the thoughts of the feminist philosopher Judith Butler and her idea of performativity. This idea broke the biological and naturalistic myths about sex which opened the assumption that sex is the cause of gender and it is that which serves as an implication for its being fluid. According to Butler, the terms "gender is performed" should be differentiated from "gender is performative." The former concept is based on the matrixes that society dictates and imposes which then becomes a guideline on how to perform a gender; on the other hand, the second concept is shaped in view of the repeated performance of gender as a subversive break towards reconstruction coming from the imposed matrixes. This paper discovered how a variety of lesbian images were formed in the two abovementioned films, wherein due to the actions and decisions of the lesbian characters, they have proved that it was only the society's matrix that control and create conflict to disrupt the free formation of these images. Although Butler is a western thinker, she was able to deepen the discourses of the Filipino scholars about gender which a number of them have endeavored to understand through gender studies and lesbian films in the country. By trying to find an alternative
\end{abstract}

(c) 2016 Judith R. Angeles, Tracie Kathlynne B. Bacarro,

F.P.A. Demeterio, III, Jonathan Vergara Geronimo, and Patricia Bettina L. Peliño

http://www.kritike.org/journal/issue_19/angeles_et al_december2016.pdf

ISSN 1908-7330 
analysis of the state of the lesbian in the Philippines that does not detach itself from the narrative of the country's patriarchal and third world status, this paper may significantly contribute to gender activism and advocating equal aspirations for a true, free society.

Keywords: Butler, lesbianism, gender performativity, independent films

\section{Introduksyon}

a konteksto ng pang-akademikong pananaliksik at pulitikal na usapusapan tungkol sa kasarian sa Pilipinas, mas malimit napagtutuunang pansin ang mga paksa ng pagkababae at pagkabakla kaysa pagkalesbiyan ${ }^{1}$. Kaya hangad ng papel na ito na makapag-ambag sa lokal na literatura at diskusyon tungkol sa nasabing penomenon sa pamamagitan ng pagsusuri sa dalawang Pilipinong "indie" (independent) film na tahasang tumalakay sa parehong penomenon. Para maging sistematiko at organisado ang pagsuri ng papel na ito ginamit nito ang kaisipan ng Amerikanong pilosopo, feminista at aktibistang si Judith Butler (ipinanganak: 1956), partikular na ang kanyang teorya tungkol sa gender bilang performativity.

Ang indie film sa Pilipinas ay umusbong sa ika-21 na daang taon sa pagitan ng paghina ng industriya ng pelikula sa bansa, dulot ng dominasyon at hegemonya ng Hollywood, at ng paglaganap ng digital na teknolohiya na lubusang nagpababa sa halaga ng produksyon ng pelikula sa antas na abotkaya na ng maliliit at independyenteng filmmaker. Itinayo ang Experimental Cinema of the Philippines (ECP) ng diktaduryang Marcos noong 1982 upang hindi lubusang malantad ang panunupil nito. Nagpondo ito ng mga pelikulang de-kalidad, at mga obrang ibinunga ay gamit na pantapal sa mga pilat ng panunupil sa industriya ${ }^{2}$. Sa ganitong konteksto, ipinalalagay na nabuo ang pormula ng independent cinema nang itayo ang nasabing ECP dahil na rin sa posturang avant-garde na nalilikha sa mga pelikulang produkto nito.

Pinasimulan ng ECP ang pagtatayo ng mga arkibong pampelikula para sa lokal na industriya, naipakilala ang sensura sa porma ng "film rating" at itinaguyod ang alternatibong pelikula. Binigyang-kahulugan ang

1 Sharon Anne Pangilinan. "Ang Pagdaloy sa Kasaysayan at Kasaysayan ng Pagdaloy ng Panitikang Lesbiyana ng Pilipinas," in Likhaan: The Journal of Contemporary Philippine Literature, 3 (2009), 217-231.

${ }^{2}$ Bienvenido Lumbera, "Ang Pelikula sa Lipunang Filipino, Ang Lipunang Filipino sa Pelikula," in Writing the Nation/Pag-akda ng Bansa (Quezon City: University of the Philippines Press, 2010), 388.

(c) 2016 Judith R. Angeles, Tracie Kathlynne B. Bacarro, F.P.A. Demeterio, III, Jonathan Vergara Geronimo, and Patricia Bettina L. Peliño http://www.kritike.org/journal/issue 19/angeles et al december2016.pdf 


\section{PERFORMATIVITY AT PAGKALESBIYAN}

alternatibong pelikula bilang pagkawala sa estetikang komersiyal at malaong nagpanihubog din sa nilalaman tungo sa pagtalakay ng mga isyung panlipunan. Sa pagsasantabi sa mga higanteng prodyuser, naisantabi rin ang kanilang kapitalistang interes na dati ay humadlang sa masining at malikhaing imahinasyon ng mga Pilipinong filmmaker. Sa paglaya ng mga Pilipinong filmmaker sa pakikialam ng mga higanteng prodyuser, tinalakay nila ang mga paksang dati ay hindi maaaring talakayin o hindi masyadong natalakay sa pelikula. Kaya naman nagkaroon ng pagkakataon ang paksa ng pagkalesbiyan na mailagay sa harap ng kamera. ${ }^{3}$

Ngunit, kung babalikan ang pagsasakasaysayan ng pelikulang lesbiyan sa Pilipinas, nagsimulang magkaroon ng espasyo ng representasyon ang mga homosekswal noong dekada '70, panahong itinuturing na gintong panahon ng pelikulang pambansa. Tinalakay sa artikulo ni Libay Cantor, na isa sa unang nagdalumat sa pelikulang lesbiyanismo sa Pilipinas, na bagaman may mga pagtatangkang lumikha ng mga lesbiyanang karakter sa mga pelikulang Filipino ay "napakababaw at napakabaluktot ng ideya ng lesbi[y]anismo ang ipinapakita sa karamihan sa mga pelikula"4. Para kay Cantor, ang diskurso sa mga naunang pelikulang homosekwal, na ang pagkalesbiyan bilang paggaya lamang sa kalalakihan at walang malay na pagpili, "pagkalito" o itinuturing na isang "phase" lamang ng pagiging babae. Sa obserbasyon naman ni Guieb tungkol sa pelikulang lesbiyan na nalikha ng pelikulang komersiyal sa mga unang panahon nito, ipinalalagay niyang walang maihahanay na matinong pelikulang lesbiyan at kung mayroon man ay komedi ang naging dulog dito. ${ }^{5}$. Sa pagpasok ng indie film at tradisyong alternatibo sa paglikha ng pelikula, binigyang-hugis ang pagtatanghal sa mga lesbiyan sa mas realistikong imahe at karakter nito.

Sa dinami-rami ng indie film na nagawa mula noong bungad ng ika21 daang taon, susuriin lamang ng papel na ito ang dalawang indie film na tahasang tumalakay sa nasabi nang paksa ukol sa pagkalesbiyan: ang Rome and Juliet ng 2006 at Ang Huling Cha-Cha ni Anita ng 2013 na binigyang-tuon at napili dahil sa baryasyon ng pagtalakay na ibinubukas ng dalawang

\footnotetext{
${ }^{3}$ Grace Javier Alfonso, "Rome and Juliet: No Stereotyping of Women who Love Women," in Manunuri ng Pelkiulang Pilipino (30 October 2014), $<\underline{\text { http://www.manunuri.com/reviews/rome_and_juliet_no_stereotyping_of_women_who_love }}$ women>, 12 March 2016.

${ }^{4}$ Olivia Cantor, "Lesbiana, Lesbiana, Paano Ka Isinasapelikula: Ilang Pagmumuni sa Pagsasadula ng mga Lesbiana sa Pelikulang Filipino," in Tabi-tabi sa Pagsasantabi: Kritikal na Tala ng mga Lesbiana at Bakla sa Sining, Kultura, at Wika, ed. by Eugene Y. Evasco, Roselle V. Pineda, and Rommel B. Rodriguez (Quezon City: University of the Philippines Press, 2003), 367.

${ }^{5}$ Eulalio Guieb III, “Mga Anyo ng Sineng Filipino: Paghahanay at Paglilinaw ng mga Kinagisnan at mga Tunguhin," in Sining ng Sineng Filipino (Quezon City: UP Sentro ng Wikang Filipino, 2009), 65.
}

(C) 2016 Judith R. Angeles, Tracie Kathlynne B. Bacarro,

F.P.A. Demeterio, III, Jonathan Vergara Geronimo, and Patricia Bettina L. Peliño http://www.kritike.org/journal/issue 19/angeles et al december2016.pdf ISSN 1908-7330

(c) $)$ BY-NC-ND 
pelikula bilang diskurso sa pangkasariang karanasan at paglikha ng representatibong imahe sa sinusuring penomena at panlipunang kalagayan.

\section{Dalawang Piling Indie Film}

Rome and Juliet: Nilikha ang pelikulang ito ni Connie Macatuno, sa ilalim ng Cinema One Originals, batay sa kwentong ginawa niya at screenplay na isinulat niya kasama si Chris Violago. Ginampanan ito ng mga kilalang artista, tulad nina Andrea del Rosario, Mylene Dizon, Rafael Rosell, Tessie Tomas at Glydel Mercado. Si Juliet (del Rosario) ay isang pre-school teacher na nakatakdang ikasal sa isang batang politikong si Marc (Rosell). Bilang bahagi ng paghahanda sa nalalapit na kasal, kinuha nila si Rome (Dizon) bilang wedding planner. Sa palagiang pag-uusap at pagsasama nagsimulang magkalapit ng loob sina Rome at Juliet sa isa't isa. Sumibol ang kakaibang damdamin na noon lamang nila naramdaman. Sa simula, hindi nila lubusang nababatid ang kanilang damdamin sa bawat isa subalit kalaunan ay unti-unti na nilang napagtanto sa kanilang mga sarili na higit pa sa magkaibigan ang kanilang nararamdaman para sa isa't isa. Minsang hinanap ni Marc si Juliet sa bahay nina Rome, naabutan niyang sabay na naliligo habang naghaharutan sina Juliet at Rome. Doon lamang niya nadiskubre na ang kanyang kasintahan ay may relasyon na kapwa babae. Matapos ang komprontasyon, nagawa pa ring patawarin ni Marc si Juliet. Subalit kinaumagahan, kinansela rin ni Marc ang kasalan dahil sa nabasa niya sa diary ni Juliet na naglalaman ng kanyang mga tula. Napabalita ang naudlot na kasal at ang dahilan nito na naging sanhi naman sa pagkaatake sa puso ng tatay ni Juliet. Sinisi ng Nanay (Tomas) ni Juliet ang naging desisyon ng kanyang anak. Hirap ang kalooban ni Juliet dahil sa pagkamatay ng ama, pagkagaling sa sementeryo, si Juliet ay nasagasaan at nawalan ng malay ng tatlong linggo. Dahil sa nangyari, natanggap ng ina ni Juliet si Rome dahil sa ipinakita nitong pagmamahal at pag-aalaga sa kanyang anak habang nakaratay sa ospital. Maging si Marc ay nagpaubaya na rin dahil alam niya sa kanyang sarili na hindi na siya mahal ng kanyang kasintahan.

Ang Huling Cha-Cha ni Anita: Nilikha ang pelikulang ito ni Sigrid Andrea Bernardo, sa ilalim ng CineFilipino, Pixeleyes Multimedia at Ekweytormc, at batay sa kwento at screenplay na siya rin ang gumawa. Ginampanan ito ng mga artista na pinangunahan nila Angel Aquino, Teri Malvar, Jay Bordon, Marcus Madrigal at Lui Manansala. Si Anita (Bordon) ay isang opisyal ng Hukbong Sandatahan ng Pilipinas na magbabakasyon sa kanilang baryo sa Obando, Bulakan. Sa kanyang paghahandang bumalik sa lugar ng kanyang kabataan, nanumbalik rin ang mga alaala nang nakalipas. Ang batang Anita (Malvar) ay napalapit noon sa kababalik lamang na si Pilar

(c) 2016 Judith R. Angeles, Tracie Kathlynne B. Bacarro, F.P.A. Demeterio, III, Jonathan Vergara Geronimo, and Patricia Bettina L. Peliño http://www.kritike.org/journal/issue 19/angeles et al december2016.pdf 
(Aquino). Sa mga mata ni Pilar isang nakababatang kaibigan lamang si Anita, ngunit sa mga mata ni Anita, si Pilar ay ang babaeng labis niyang hinahangaan at naging daan sa paggalugad ng tunay nitong sekswalidad. Isa sa mga dahilan sa pagbalik ni Pilar sa kanilang baryo ay si Oscar (Madrigal), dati niyang kasintahan at pinsan naman ni Anita. Labis na nasaktan si Anita nang madiskubre niya ang lihim na relasyon nina Pilar at Oscar. Nagbalik ang damdamin para sa isa't isa ng dating magkasintahan ngunit muling naudlot ang relasyon dahil buntis na ang kasalukuyang kasintahan ni Oscar. Dahil sa nangyari, uminom ng pampalaglag si Pilar. Isang araw, sinundan ni Anita si Pilar sa bahay nito. Nakita niyang nawalan ng malay si Pilar habang papasok ng bahay. Tinulungan ni Anita si Pilar hanggang sa bumuti ang pakiramdam ng huli. Bilang kapalit, binigyan ng libreng masahe ni Pilar si Anita kahit pa tumanggi ang una dahil sa kulang pa ang kanyang naipong pera bilang kabayaran sa hilot. Sinabi ni Pilar kay Anita ang lihim nito at dahilan ng paglayo sa kanilang baryo ay dahil sa panghahalay ng sariling ama. Paggising ni Anita ay wala na si Pilar. Nagtapos ang pelikula sa aktwal na pagdalaw ni Anita sa kanilang baryo at sa kanyang muling pagbisita sa kapistahan ni Santa Clara.

\section{Gender bilang Performativity: Mga Imahe at Panlipunang Paghubog ng Pagkalesbiyan sa Dalawang Pelikula}

Si Judith Butler ay kilalang teorista sa larangan ng feminismo at pagaaral tungkol sa kasarian. Kaugnay sa interes at adbokasiya ng kanyang mga pag-aaral, mahalagang malaman ng mga mambabasa na si Butler ay isang lesbiyan, na may kinakasamang babaeng partner, at mayroon silang anak na lalaki.

Naging laganap sa maraming teorista ukol sa kasarian ang pagbibigay-diin sa pagkakaiba ng sex, bilang bayolohikal at natural na katangian, at gender, bilang kultural na katangian. Parehong "kasarian" ang salin sa Filipino ng "sex" at "gender," kaya mula sa puntong ito ay isantabi na natin ang salitang "kasarian" at gamitin na lamang ang Ingles na mga kategoryang "sex" at "gender" para sa politikal na operasyon nito sa kasalukuyang pag-aaral. Sang-ayon si Butler sa laganap na prinsipyong ang gender ay isa ngang kultural na katangian. ${ }^{6}$ Ngunit tutol siya sa laganap rin na prinsipyong ang sex ay isang bayolohikal at natural na katangian. Ipinaliwanag ni Butler na kapag tinanggap natin ang pagiging bayolohikal at natural ng sex, o ang pagiging babae at pagiging lalaki, mapipilitan tayong

\footnotetext{
Routledge, 1990).

(C) 2016 Judith R. Angeles, Tracie Kathlynne B. Bacarro,

F.P.A. Demeterio, III, Jonathan Vergara Geronimo, and Patricia Bettina L. Peliño http://www.kritike.org/journal/issue 19/angeles et al december2016.pdf ISSN 1908-7330
}

${ }^{6}$ Judith Butler, Gender Trouble: Feminism and the Subversion of Identity (New York:

(c) BY-NC-ND 
tanggapin din ang implikasyon na dahil babae ang isang tao dapat lamang na pambabae ang kanyang gender, o dahil lalaki ang isang tao dapat lamang na panlalaki ang kanyang gender. Konseptwal na pinag-iiba naman ng Pilipinong iskolar na si Eulalio R. Guieb III, ang dalawang termino batay sa karanasang patriyarkal sa Pilipinas, aniya: "Kung gayon, bagama't dalawa lang sa usapin ng ba[i]yolohiya ang sex ng tao (babae at lalaki), maraming socially constructed na kasarian, o gender preference o kinikilingang disposisyong pangkatauhan: lalaki, babae, bisexual, lesbian, transvestite, transgender, at iba pa." Kung susuriin, nagkakasundo sina Butler at Guieb na hindi maikakahon ang konsepto ng gender lalo at pag-uusapan ang kompleks ng hegemonikong diskursong patriyarkal sa lipunan.

Sa pelikulang Rome and Juliet, masusuri ang imahe ng hindi malay na pagkalesbiyan sa dalawang pangunahing karakter. Ilan sa mga imaheng nakita, una, pagkaramdam ng selos ni Juliet para kay Rome. Sa kabila ng nakatakdang kasal sa nobyo ay nakaramdam pa rin ng selos si Juliet para sa dating nobyo ni Rome na naging bisita nito. Dito nagsimula ang agam-agam sa damdamin ni Juliet at maging ni Rome. Nagisnan ni Juliet na magkayakap na nakahiga sa sofa si Rome at dating nobyo nito. Nagpakita ng pagkainis ang padabog na paghahanda ng agahan ni Juliet sa nasaksihan. Nagising si Rome dahil sa ingay at pilit na inuusisa ang problema. Biniro nito nang paulit-ulit si Juliet at sinabing nagseselos daw siya na mariin naman nitong itinanggi. Dahil sa paglalapit ng mukha habang nangungulit, nagdikit ang kanilang mga labi at parang napaso ang dalawa at tumigil sa pagtatalo. Sa ganitong inilahad na mga tagpo, mauunawaan ang ideya ni Butler ukol sa konsepto ng performance na walang radikal na bahid ng pagtatanghal sa gender at hindi nagiging buo ang aktwalisasyon dahil manipestasyon lamang ng pamantayang kultural at sitwasyong nagkokontrol ang humuhubog sa intensiyon ng aktor. Ayon kay Butler na sinipi nina Oslon at Worsham, "...the meaning of the performance is established by the intention of the actor-hardly. What are being performed are the cultural norms that condition and limit the actor in the situation." 7 Kung gayon, ang praktika ng pagkalesbiyan gaya ng pagseselos at mga pahiwatig ng pagkagusto ngunit hindi lantarang maipahayag at maideklara sa malayang pagkilos ay bunga ng mga restriksyong kultural na dikta ng kumbensiyong patriyarkal, mga kondisyong naglilimita kay Juliet na hayagang ilantad ang pakiramdam na hinuhubog ng kanyang gender.

Sa kabilang banda, maipaliliwanag naman ang taliwas na konsepto ng performance sa panukalang performativity ni Butler bilang isang

\footnotetext{
${ }^{7}$ Gary A. Oslon and Lynn Worsham, “Changing the Subject: Judith Butler's Politics of Radical Resignification," in Politics of Possibility: Encountering the Radical Imagination (New York: Paradigm Publishers, 2007), 30.
}

(c) 2016 Judith R. Angeles, Tracie Kathlynne B. Bacarro, F.P.A. Demeterio, III, Jonathan Vergara Geronimo, and Patricia Bettina L. Peliño http://www.kritike.org/journal/issue 19/angeles et al december2016.pdf ISSN 1908-7330 


\section{PERFORMATIVITY AT PAGKALESBIYAN}

diskursibong bagay na may malayang katangiang hindi maikakahon sa mga pamantayang idinidikta ng lipunan. Maiuugnay ang substansya ng teoryang ito ni Butler ukol sa gender sa palagay ni Nietzche sa On the Genealogy of Morals na nagsasabing "there is no 'being' behind doing, effecting, becoming, 'the doer' is merely a fiction added to the deed - the deed is everything"8 Masusuri ang diwa ng teoryang ito sa mga imaheng nabuo naman sa karakter ni Anita, ang hayagang pagpapakita ng kawalang-hilig sa pananamit, kagamitan, at mga aktibidad na pambabae ay may malay na pagpapasya sa paulit-ulit na paggampan. Kitang-kita sa praktika ng pagkalesbiyan ni Anita na mayroon na siyang nabubuo na sarili niyang identidad na taliwas sa isteryotipong konstruksyon ng isang babae sa lipunang Pilipino. Sa unang bahagi ng pelikula, nakadamit pang-sundalo si Anita at naglalaro ng kasal-kasalan kasama ang dalawa niyang kalaro na sina Carmen at Goying. Sa larong ito, si Anita ang groom, si Carmen ang bride at si Goying ang pari. Makikita sa ganitong pagpoposisyon ang pagkiling ng karakter na si Anita sa gampaning lalaki. May pagkakataon na pinagsusuot ng bestida si Anita ng kanyang ina at dali-dali niyang pinalitan ng t-shirt na nagpapakita ng kanyang pagtanggi sa hinuhubog na katauhan ng kanyang ina sa kanyang pagkatao. Ang pagkainis sa pagsusuot ng gown at paglahok sa Santa Cruzan ay maituturing na manipestasyon din ng pagbasag sa kinokondisyon na tradisyong pambabae na hinuhubog sa lipunang ginagalawan nito. Kumikiling din si Anita sa pagbibisekleta at paglalaro ng trolly na karaniwang libangan ng lalaki. Sa mga ipinapakitang pagbalikwas ng karakter sa isteryotipong pambabaeng gawain, gampanin at kaayusan, tumitingkad ang pagpapakahulugan sa gender bilang performative, na nabubuo batay sa pagsasagawa at pagsasabuhay na higit pa sa simpleng pagpili o pagtatakda ng aktor.

Kung lalagumin ang kaibahan ng performance at performativity sa pag-aaral kay Butler, importanteng alamin ang pagkakaiba ng katagang "gender is performed" sa "gender is performative." Kapag sinabing "gender is performed," ibig sabihin nito ay nadidiktahan na ang kasarian bago pa maobserbahan ang mga kaugalian at gawain ng isang tao. Mayroon nang tiyak na kaugalian na ginagawa para masabi na ang isang tao ay isang lalaki, babae, bakla o lesbiyan. Sa kabilang panig, kapag sinabing "gender is performative," nangangahulugang ang kaugalian na ipinapakita ang may kapangyarihang magdikta kung ano ang gender ng isang tao. Kung tutuusin, parang pareho lang ang dalawang kataga, ngunit sa "gender is performative", ang gender ng isang tao ay epekto lang ng mga kaugalian niya

${ }^{8}$ Ibid., 24-25.

(C) 2016 Judith R. Angeles, Tracie Kathlynne B. Bacarro,

F.P.A. Demeterio, III, Jonathan Vergara Geronimo, and Patricia Bettina L. Peliño http://www.kritike.org/journal/issue 19/angeles et al december2016.pdf ISSN 1908-7330

(c) $)$ BY-NC-ND 
at sa una pa lamang, wala talagang gender sa kadahilanang ang kaugalian ang nagsasabi nito. Ito ay kabaliktaran naman sa "gender is performed." Halimbawa, sa bakla o bading, kapag sinabi mong bakla o bading ang isang tao, ito ay lalaking nagdadamit babae o nagpapakita ng mga kaugaliang madalas makita o maranasan ng mga babae. Kaya sa ganitong pag-iisip, mayroon nang pangunahing ideya ng pagiging "bakla" ang isang tao dahil natatag na ang gender dito. Pero para sabihin ni Butler na "gender is performative," ipinapalagay na niya na sa simula pa lamang ay wala talagang gender dahil ito ay epekto lamang ng mga kaugalian ng tao. Sa dalawang pelikula, binasag ang mga isteryotipong konseptong bitbit ng "gender performance" sa pamamagitan ng pagsisikap ng pelikulang tanggalin ang mga kategorya ng pagkalesbiyan na naitatag na sa karaniwang kamalayan ng lipunan. Kung tutuusin, hindi mamamalayan ng mga manonood ang pagpaksa sa pagkalesbiyan bilang sentrong isyu ng pelikula dahil sa simula pa'y hindi ninanais ng pelikulang gawing "subject of study" ang gender at ibukod na eksotikong pagtatanghal ito.

Batay naman sa mga ideyang binanggit ni Butler sa kanyang Gender Trouble: Feminism and the Subversion of Identity, lalong mauunawaan ang malalim na kahulugan ng performativity na nakasalig sa malalim na lebel ng panlipunang konsepto ukol sa pagiging lesbiyan. Taliwas sa karaniwang ideya ng sex at gender na ang "sex ay sanhi ng gender," ipinaglalaban ni Butler na hindi ito totoo. Sa halip ay "gender ang nananaig, at hindi sex." Kung isasakonteksto ito sa karanasang ipinapamalas sa pelikula, ang pagkakaroon ng relasyon nina Rome at Juliet na hindi naglilinaw ng takdang posisyon o gampanin sa relasyon ay nagpapakita pa rin ng imaheng "femme" sa mga karakter o pagpapanatili ng pisikal na posturang babae habang nagaganap ang transpormasyong panloob. Nangyari ito matapos na umamin si Juliet ng kanyang nararamdaman para kay Rome sa pamamagitan ng pagbabasa ng isang tula na nagpapahayag ng matinding damdamin ng karakter. Dahil dito, natuklasan nila sa kanilang sarili ang nais nilang mangyari sa kanilang dalawa. Sa puntong ito, malinaw ang pananaig ng gender o malayang pagpapasya ng kasarian at walang kaugnayang pangsanhi (causal connection) ang sex at gender sa ganitong malayang pagsasabuhay. Ayon pa kay Butler, totoo ngang may pagkakaiba ang pisikal na anyo ng babae at lalaki, ngunit kultura ang nagpapalaki sa pagkakaibang ito. Sa halip na sex ang sanhi ng gender, ipinakita niya na gender ang sanhi ng sex. ${ }^{9}$ Hindi lamang pinutol ni Butler ang ugnayang pangsanhi sa pagitan ng sex at gender, kung hindi binaligtad pa niya ito. Sa puntong pareho nang artipaktwal ang sex at gender, gaya ng nabanggit na, hindi na dapat limitahan ang konsepto ng gender sa mga kumbensiyong pambabae o

${ }^{9}$ Ibid., 30.

(c) 2016 Judith R. Angeles, Tracie Kathlynne B. Bacarro, F.P.A. Demeterio, III, Jonathan Vergara Geronimo, and Patricia Bettina L. Peliño http://www.kritike.org/journal/issue 19/angeles et al december2016.pdf ISSN 1908-7330 


\section{PERFORMATIVITY AT PAGKALESBIYAN}

panlalaki lamang. Gayundin, maaaring isakonteksto ang mga pahayag na ito sa pagpili ng isang tao ng katulad o kasalungat na gender para sa isang romantikong relasyon.

Mapatutunayan din ang diwa ng ganitong mga pananaw sa pelikula sa pamamagitan ng pagiging malapit ni Anita kay Pilar. Kung tutuusin, wala namang masama kung maging malapit ang dalawang babae sa isa't isa lalo pa't may malaking agwat ang kanilang edad at walang anumang itinatakdang pamantayan sa pagkakaibigan. Ngunit sa kasong ito, ang dahilan ng pagiging malapit ni Anita kay Pilar ay dahil sa pagkabighani nito sa dalaga sa unang pagkakataon; sinabi pa niya sa kanyang mga kaibigan na si Pilar ang nais nitong makasama, kung kaya't ang pagiging malapit niya ay hindi lamang isang simpleng pagtutumbas sa "kaibigan" dahil ang isa sa kanila ay may sikretong pagtingin at malayong pagtanaw pa sa pagiging "asawa" balang araw. Mapatutunayan din ito sa matinding pagnanais ni Anita na magpahilot kay Pilar at pag-iipon ng sapat na pera upang matamo ang inaasam na kaganapan ng nararamdaman. Malinaw na binabasag nito ang kumbensiyong naglilimita lamang sa heterosekswal na uri ng pakikipagrelasyon at tumatawid sa homosekswal at iba pang kombinasyon ng ugnayan, dahil ang pamantayang ginagamit ay ang malayang pagpili at pakiramdam.

Masusuri din ang ganitong pananaig ng gender sa mga tagpo ng hindi minsanang pagtatalik ng dalawang bidang babae sa pelikulang Rome and Juliet, matapos ang pag-amin sa kanilang nararamdan. Ang pagtatalik ng dalawang karakter bilang isang gawain ay pagpapamalas ng malalim na ugnayan at pagpapahayag ng gender na hindi malilimitahan pa ng kumbensiyong kakabit ng sex. Maibabahagi rin kaugnay sa mga tagpong ito ang diin ni Butler tungkol sa performativity, na ang gender ay hindi dahil sa sex, kung hindi isang produkto lamang ng mga tungkulin o mga bagay na ginagampanan $\mathrm{ng}$ bawat isa sa ating lipunan o buhay.

Ang imahe naman ng pakikipagrelasyon ng dalawang bidang babae sa lalaki, si Rome na iniwan ng boyfriend para magpakasal sa iba samantalang si Juliet naman na nakatakdang ikasal subalit nakadama pa rin ng pagmamahal sa parehong kasarian. Sa ganitong pagkakabuo, maaring ipalagay ang nosyong hindi maikakahon ang gender sa mga kategoryang ipinapataw ng lipunan gaya ng lesbiyan na maaaring bisekswal at/o babae. Muling mapagnilalayan sa mga karanasang ito ang diwa ng pagiging "fluid" ng gender. Ang pagbaklas ni Butler sa bayolohikal at naturalistikong mito tungkol sa sex ay nagbukas sa posibilidad na maaaring hindi lamang dadalawa ang sex, at ang kanyang pagtanggi na sanhi ang sex sa gender ay nagbigay implikasyon na hindi rin dadalawa o aapat lamang ang gender. Sa

(C) 2016 Judith R. Angeles, Tracie Kathlynne B. Bacarro,

F.P.A. Demeterio, III, Jonathan Vergara Geronimo, and Patricia Bettina L. Peliño http://www.kritike.org/journal/issue 19/angeles et al december2016.pdf ISSN 1908-7330

(cc) BY-NC-ND 
katunayan, naniniwala si Butler na marami ang gender at hindi lamang ito pambabae, panlalaki, pagkabakla at pagkalesbiyan.

Nahuhubog din ang gender bilang performative sa kabila ng mga panlipunang pagpigil at pagkontol na lumilikha ng mga epekto ng pagbabawal, pagsasantabi at pagbabanta ng kaparusahan sa hindi pagsunod sa pamantayan at paulit-ulit na pagsasagawa ng gender na maaaring magdulot ng pagbuo at pagkawasak nito. Halimbawa, maaaring may nalilikhang takot sa loob ng isang kloset kung bakit hindi mailantad ang ganap na pagsasagawa ng kanyang gender. Sa pelikula, matingkad na napalitaw ang mga social matrix na bumubuo ng kontrol at pagbabanta. Bilang tugon sa ganitong social matrix, pinatingkad sa pelikula ang imahe ng pagnanais ng dalawa na ipaglaban ang relasyon. Makikita ito sa dulong bahagi ng pelikula na marami ang tumutol at nanghusga sa uri ng relasyong umiiral sa dalawa. Naging sanhi ito ng maraming suliranin lalo sa panig ni Juliet. Itinakwil siya ng kanyang ina dahil na rin sa pagkamatay ng kanyang ama, nagalit ang kanyang dating nobyo, pinag-tsitsismisan siya ng buong baryo at napipinto pang matanggal sa trabaho dahil sa kanyang gender. Magkagayunman, pinili pa rin nilang manatili sa piling ng bawat isa at patunayan na tunay ang kanilang pagmamahal sa kabila ng magkatulad na gender, ang paulit-ulit na praktika ng kanilang nararamdaman. Nailarawan sa pelikula ang iba't ibang panlipunang pwersa na nagdidikta ng mga isteryotipo at de-kahong pagtingin sa gender na iniaangkop alinsunod sa mga gampaning domestiko, kultural at ekonomiko. May namamayaning panlipunang matrix na paulit-ulit na nagdidikta ng unipormidad sa gender na naglilimita lamang sa pagkalalaki at pagkababae, ngunit dahil hindi maikakahon ang gender, palagiang nariyan ang paglaban.

Tinalakay din ni Butler ang naturalistiko at daynamikong praktika ng gender na: "Gender ought not to be construed as a stable identity or locus of agency from which various acts follow; rather, gender is an identity tenuously constituted in time, instituted in an exterior space through stylized repetition of acts."10 Sa pahayag na ito, ang kahulugan ng time ay hindi tumutukoy sa panahong nangyayari makalipas ang isang gabi lamang. Ang konsepto ng oras sa puntong ito ay ang paulit-ulit na kilos na nagpapahiwatig din na hindi maikukulong sa panahon ang pagsasakilos ng gender. Gayundin, sa exterior space, na importanteng alalahanin na pupwedeng mag-iba ang konsepto ng gender depende kung saan naninirahan ang isang tao. Sa pagkakabuo ng imahe ni Anita halimbawa, makikita ang manipestasyon ng pagkalesbiyan sa palagiang pamamantasya kay Pilar sa iba't ibang paraan at pagkakataon: ang ilusyon na kasabay si Pilar sa trolly, pagtabi sa kanyang higaan, paghalik sa

${ }^{10}$ Ibid., 179.

(c) 2016 Judith R. Angeles, Tracie Kathlynne B. Bacarro, F.P.A. Demeterio, III, Jonathan Vergara Geronimo, and Patricia Bettina L. Peliño http://www.kritike.org/journal/issue 19/angeles et al december2016.pdf ISSN 1908-7330 


\section{PERFORMATIVITY AT PAGKALESBIYAN}

kanya sa pamamagitan ng silindro, pagguhit ng mukha ng isang babae sa unan, pagputol sa kanyang mahabang buhok ni Pilar, at pakikipagsayaw ng Cha-Cha sa dalaga ay likha ng lahat ng kanyang hindi maipahayag na damdamin at praktis ng gender. Mula sa simpleng pamamantasya nalinang ang pagnanasa tungong realistikong pagsasagawa ng gender gaya ng panliligaw sa pamamagitan ng paghahatid sa palengke kay Pilar at pagdalaw sa bahay pagkatapos ng oras ng panghihilot ni Pilar. Mapatutunayan sa ganitong mga tagpo ng paulit-ulit na pamamantasya ang paglampas sa limitadong oras at espasyo na pinagsimulan din ng aktwalisasyon at kaganapan ng gender.

Ang paulit-ulit na pagsasagawa ng gender labas sa de-kahong pananaw at hindi pakikipagkompromiso sa mga nilikhang pamantayan ay politikal na pagpapasyang pagmumulan ng paglaban, subersyon at paglaya. Kailangang hamunin ang kultural na matrix na may sinusunod na kaayusang nagdidikta ng katanggap-tanggap na identidad ng gender dahil sa pagtugon nito sa umiiral na mga kultural na pamantayan at kaayusan. Sa dalawang pelikula, makikita ang magkakaibang pamamaraan ng mga lesbiyan sa pagtatanghal ng kanilang pagkatao at paglaban sa ipinapataw na kaayusan ng mga umiiral na social matrix. Ang pagsasabi ni Anita ng kanyang nararamdaman para kay Pilar. Sa isang bahagi ng pelikula, makikitang tila may inaamin si Anita ukol sa dahilan ng kanyang pagkagusto kay Pilar lalong-lalo na ang pisikal na katangian nito. Gayundin, makikita ito sa matapang na pagtatapat sa kanyang pamilya sa pamamagitan ng kanyang pinsan na si Oscar at ng kanyang ina. Patunay ito ng transpormasyon ng nararamdaman ni Anita na desidido sa kanyang pagpili at pagpapasya, isang politikal na praktika ng kanyang gender at deklarasyon ng identidad. Sa kabilang banda naman, ang mga nabanggit nang pakikipagtunggali nina Rome at Juliet para ipahayag ang kanilang nararamdaman sa kabila ng mga panlipunang kontrol at banta ay malinaw na nagpapamalas naman ng pagsasakapangyarihan sa kanila

\section{Paglaban, Pagbalikwas at Paglaya: Muling Pagbubuo ng Pagkalesbiyan at Tunggaliang Panlipunan}

Sa kabila ng pag-iral ng mga panlipunang pwersa na nagdidikta at naglilimita sa unipormidad ng gender na nakatali sa kategoryang heterosekswal bilang batayan ng pag-iral, nalilikha ang kahulugan ng gender bilang isang kontra-diskurso at kontra-kulturang pagtatanghal sa isang patriyarkal na lipunan. Sa dalawang pelikula, masasalamin ang mga namamayaning tunggaliang ito sa panig ng nagpapataw ng matrix ng

(C) 2016 Judith R. Angeles, Tracie Kathlynne B. Bacarro,

F.P.A. Demeterio, III, Jonathan Vergara Geronimo, and Patricia Bettina L. Peliño http://www.kritike.org/journal/issue 19/angeles et al december2016.pdf ISSN 1908-7330

(c) $)$ BY-NC-ND 
lipunan at panig ng isinasantabi at humahamon sa umiiral na kaayusan. Masasalamin din ang mga tunggaliang ito na maikakategorya sa mahahalagang institusyon/pwersang panlipunan na nagtatakda ng panlipunang matrix gaya ng estado, simbahan at akademya. Gayundin, matutunghayan sa pelikula ang iba't ibang pamamaraan ng paglaban ng mga karakter na lesbiyan upang itanghal ang isinasantabing pag-iral.

Sa pelikulang Rome and Juliet, ang unang paraan ng pagkontrol ay sa pamamagitan ng diskriminasyon. Unang nakaranas ng hindi pagtanggap sa kanyang gender ang ina ni Juliet. Sinaktan nito si Juliet sa pisikal at pasalitang paraan gaya ng pananampal, paninisi, at panghuhusga sa kanyang pakikipagrelasyon at pagyurak sa pagkatao ng kanyang karelasyon. Sa ganitong punto, ipinapakita lamang ng pelikula ang realistiko at karaniwang reaksyon ng isang ina na nagpapalaki ng anak sa isang konserbatibong tipo ng lipunan na may luma at tradisyunal na pananaw. Ang ganitong pagkakahon ng ina ni Juliet sa kanyang gender ay taliwas sa pagtingin ni Butler sa gender, para sa kanya, pareho nang artipaktwal ang sex at gender kaya dapat ay wala nang magsasabi na dahil babae ang isang tao ay kailangang pambabae ang kanyang gender, o dahil lalaki ang isang tao ay kailangang panlalaki ang kanyang gender. Bilang isang anak, tinanggap na lamang ni Juliet ang pagtatakwil ng kanyang ina ngunit bilang pagliligtas sa kanyang pagpapasya ay ipinagpatuloy ang kanyang pakikipagrelasyon bilang praktis ng kanyang gender. Kaugnay rin sa uri ng diskriminasyon ang aspektong ekonomiko at pamantayang ipinapataw sa larangan ng propesyon, dahil sa kumakalat na tsismis ukol kay Juliet, kinausap siya ng kanyang prinsipal sa pinapasukang pre-school at hinatulan ng administrasyon ang kanyang kagalingan bilang guro batay sa kanyang gender. Masusuring hindi patas ang batas ng empleyo pagdating sa mga homosekswal. Sa kabila ng pagiging mahusay na guro ni Juliet ay mawawalan siya ng trabaho dahil lamang sa pagiging lesbiyan. Ang hindi pagsang-ayon ng mga magulang ng mga estudyante sa eskwelahan ay pagpapakita rin ng namamayaning homopobikong kamalayan ng mga mamamayan. Bilang tugon sa mga paraan ng pagpigil, pagkontrol at pagbabanta, at takot na naranasan ni Juliet ay itinuloy pa rin niya ang kanyang pasya na maging lesbiyan at pinanindigang huwag ipagpatuloy ang kanyang kasal kay Marc. Mahalagang maitala ang reaksyon ni Marc na sa una ay hindi tanggap ang pagiging lesbiyan ng nobya subalit kalauna'y naintindihan at natanggap niya ang gender nito. Naging malaking usapin para kay Marc ang pakikipagrelasyon ni Juliet kay Rome dahil ito ay isang babae. Gayundin, makabuluhan ang ginamit na metapora ng pelikula ukol sa pagdidikta ni Marc kay Juliet sa pamamagitan ng pagbibigay ng panali sa buhok at pagbubutones ng damit na nagpapahiwatig ng namamayani pa ring 


\section{PERFORMATIVITY AT PAGKALESBIYAN}

estrukturang pyudal kung paano dapat kumilos ang isang babae sa isang konserbatibo at patriyarkal lipunan.

Sa pelikulang Ang Huling Cha-Cha ni Anita, ang pagtatakda ng kultural na pamantayang kumbensyunal ay nagtatakda ng mga inaasahang gawi at pagkilos na pilit ipinapataw ng ina sa batang Anita. Bilang subersibong tugon ng batang Anita sa pamantayang ito, taliwas ang kanyang inaasal at ikinikilos sa ipinapataw na pamantayan ng ina, ni hindi nagkaroon ng alinlangan ang batang Anita sa kanyang gender na lalong napagtibay sa pagdating ni Pilar. Ang pagiging isang relihiyoso at panatiko ng ina ng batang Anita ang ipinapalagay na isa sa malakas na pwersang humuhubog ng kanyang sarado at isteryotipong pananaw sa gender. Itinatanghal sa pelikula ang kapangyarihang taglay ng simbahan sa pagpapalaganap ng ritwalistikong paniniwala na lumilikha ng mga kultural na pamantayan sa gawi at pagkilos ng mga sakop nito. Matingkad na kontra-dikursong argumento ng pelikula ang pagiging lesbiyan ng batang Anita sa kabila ng ipinapalagay na produkto ng pamamanata kay Santa Clara. Nais igiit ng ganitong naratibo ang paglalagay ng espasyo sa mga homosekswal at pagangkin sa kanila bilang mga nilikha rin ng Diyos. Masasabing ang paulit-ulit na pagtanggi ng batang Anita sa unipormidad na ipinapataw sa pananamit at gawi ay malinaw na isang anyo ng subersyon at pagbalikwas sa hegemonikong tradisyong konserbatibo sa pagbibihis at pagpapakahulugan sa kanyang gender na hindi maikakahon o maididikta ninuman. Ang paghuhugis ng kamalayan ni Anita batay sa tinatanaw na uri ng pakikipagrelasyon ay masasalamin naman sa deklarasyon ng kanyang katauhan sa mga kalaro na nagpapakita rin ng paggigiit at malayang pagpili. Dahil nasa hukbong sandatahan naman ang matandang Anita (Bordon). Hindi naging balakid ang kanyang napiling gender sa kanyang napiling propesyon, lalo pa't matingkad naman ang machismong oryentasyon sa larangang militar. Maliit lang din ang naging bahagi sa pelikula ng matandang Anita subalit nagsisilbing balidasyon ito ng kanyang kaganapan na tinahi ng kanyang musmos na gunita at nakaraan.

\section{Kongklusyon}

Mula sa pagsusuring ginawa sa dalawang pelikula, nagkakaroon ng matibay na pundasyon ang kataga ni Butler na "Gender is performative." Bagaman siya ay hindi isang Pilipinong pilosopo, naging akma ang kanyang pananaw ukol sa pagpapakahulugan sa gender maging dito sa Pilipinas na may matinding karanasang partriyarkal, isang dominanteng diskursong pangkasarian na mahigpit ding tinutunggali ni Butler sa kanyang mga teorya

(C) 2016 Judith R. Angeles, Tracie Kathlynne B. Bacarro,

F.P.A. Demeterio, III, Jonathan Vergara Geronimo, and Patricia Bettina L. Peliño

http://www.kritike.org/journal/issue 19/angeles et al december2016.pdf

ISSN 1908-7330

(c) $)$ BY-NC-ND 
at maaaring maging sa praktikang panlipunan bilang lesbiyan. Kung palalalimin pa ang argumentong ito sa isyung pangkasariang kaugat sa hustisyang panlipunan, maaaring pagnilayan ang mga pahayag ni Guieb na: “...ang patriyarka sa kabuuan ay tungkol sa pagsusumikap ng kapwa babae at lalaki tungo sa isang uri ng katarungang panlipunan (social justice) na magpapalawak sa kani-kanilang opsiyon sa lipunan nang walang kasangkot na pang-aapi sa kap[u]wa lalaki at babae." 11 Ang teorya ni Butler ukol sa pagbubuo ng gender batay sa mga itinatakdang panlipunang matrix at pagwasak nito sa pamamagitan ng paghamon at paglaban gamit ang paulitulit na pagtatanghal bilang proseso ng subersiyon ay maituturing na matingkad na karanasan ng pakikibakang pangkasarian saanmang panig ng daigdig. Bagaman, hindi isinasantabi ng ganitong obserbasyon ang katotohanang magkakaiba ang anyo ng pagkontrol at pamamaraan ng paglaban na isinasagawa sa iba't ibang bansa ay masasabing relatibo namang magkaugnay ang mga lipunan sa daigdig na kapwa dumaranas ng pagsasantabi at pagsikil sa malayang pagpili ng gender at pangangailangan ng pambansa at pandaigdigang kilusan para sa panlipunang pagkakapantaypantay. Kung isasakonteksto ang pag-unawa sa kabuluhan ng pag-aaral sa gender na kabuhol ng panlipunang karanasan ng mga bansa partikular sa ikatlong daigdig, mahalagang mailapat bilang kaugnay ang panunuri ni Tolentino ukol sa isyu feminisasyon ng pagkalalaki at paghubog ng mga panlipunang entidad na kabuhol ng proyektong service economy sa Pilipinas sa edad ng globalisasyon. ${ }^{12}$ Napalalim ni Butler ang diskursong Pilipino ukol sa gender, sa pamamagitan ng paghahapag ng alternatibong pagsusuri sa kalagayan ng lesbiyan sa Pilipinas na hindi bukod sa politikal na kontekstong patriyarkal at pagiging kasapi ng ikatlong daigdig nito.

Sa tulong ng ilang indie film, na kadalasan ay mas bukas sa mga temang ganito, naipakita na tunay ngang ang paulit-ulit na gawain at kaugalian ang siyang nagiging batayan ng pagtatalaga sa isang tao bilang lesbiyan, bakla, tomboy, bisekswal at iba pang kategorya. Sa dalawang pelikulang ginamit sa pagsusuring ito, hindi batayan ang edad upang magpakita ng mga gawi at kaugalian na magtatakda sa gender ng isang tao dahil kung titingnang mabuti, magkaiba ang edad, antas at paraan ng pagtatanghal sa pinaksang mga lesbiyan. Napatunayang sumisibol ang pagkalesbiyan na walang pinipiling edad at panahon na nagsasantabi sa nosyong bayolohikal at kultural ang pagkakahubog ng gender. Ang

${ }^{11}$ Ibid., 63.

12 Rolando B. Tolentino. “Lalaking Pin-up, GRO, at Macho Dancer" In Lalaking Pin-up, GRO, at Macho Dancer: Ang Pagtatanghal ng Pagkalalaki sa Edad ng Globalisasyon (Pasig City: Anvil Publishing, Inc., 2004), 1-48.

(c) 2016 Judith R. Angeles, Tracie Kathlynne B. Bacarro, F.P.A. Demeterio, III, Jonathan Vergara Geronimo, and Patricia Bettina L. Peliño http://www.kritike.org/journal/issue 19/angeles et al december2016.pdf 


\section{PERFORMATIVITY AT PAGKALESBIYAN}

dalawang pelikula ay may magkaibang pagtatanghal ng gender. Sa Rome and Juliet, hindi binibigyan ng tiyak na leybel ang ginagampanan ng mga lesbiyanang karakter kung sinong gumaganap sa babae at lalaki samantalang malinaw ang posisyon ng ikalawang pelikula sa pagtatanghal kay Anita bilang nagdadalagang lesbiyana na umibig sa nakatatandang babae. Natuklasan sa papel na ito na ang pagkakabuo sa mga imahe ng lesbiyan sa dalawang pelikula ay ibinunga ng kanilang paulit-ulit na pagkilos at pagpapasya na halos walang ipinagkaiba sa heterosekswal na gender: (1) imaheng femme o pagpapanatili ng posturang babae, (2) pakikipagtalik bilang praktika ng pagnanasang sekswal, (3) pagtaliwas sa isteryotipong unipormidad ng pananamit, kagamitan at aktibidad, at (4) mapangahas na pakikipaglaban at deklarasyon ng pag-ibig. Samantala, may mga natuklasang naiibang praktis ng pagkalesbiyan bilang gender: (1) pagkagusto sa parehong sex at (2) pagpapantasya o pag-iilusyon sa malayang sarili. Kung susuriin, hindi naman mailalayo ang praktis ng gender maging heterosekswal man o homosekswal man ito, tanging ang matrix ng lipunan ang nagpapataw ng pamantayan na nagsisilbing dikta at kontrol upang lumikha ng tunggalian sa malayang pagkakabuo nito.

Kung susuriin, walang nabanggit sa pelikula na anumang leybel upang ilarawan ang gampanin/role at pagkilos ng mga karakter sa pelikula. Magkaiba rin ang paraan at tindi ng pagkontrol sa mga lesbiyan sa dalawang pelikula: 1) ang pagkontrol ng pamilya na may konserbatibong pananaw na hinuhubog ng kamalayang panatiko at relihiyoso; 2) isteryotipong konstruksyon sa larangan ng propesyon; 3) matinding homopobikong kamalayan lalo sa pook-rural; at 4) namamayaning pamantayang kultural na isinasabuhay sa isang lipunan.

Napatunayang ang matrix ng lipunan din ang nagtatakda ng kawalan ng patas na pakikitungo sa mga lesbiyan na madalas na nakakaranas ng diskriminasyon dahil itinuturing na naiiba at taliwas sa normatibong kaayusan. Bagaman, matingkad din na masusuri na kumpara sa iba pang tipo ng homosekswal, ipinapakita sa dalawang pelikula na madaling tanggapin ang mga lesbiyan sa ating makapamilya at machismong kulturang Pilipino na pinatunayan sa parehong pelikula. Natanggap ng ina ni Juliet ang kanyang pagkalesbiyan gayundin ang ina ni Anita sa murang gulang pa lamang nito.

Sa kabila ng pagtatanghal sa mga lesbiyan bilang marhinalisadong sektor ng lipunan, nagbubukas ng politikal na potensyal ang dalawang pelikula na igiit ang diskurso ng humanisasyon sa mga lesbiyan sa lipunang Pilipino. Bagaman, marapat nga na lampas pa sa ganitong makitid na diskurso ay marapat na maitanghal nang ganap ang mga homosekswal

(C) 2016 Judith R. Angeles, Tracie Kathlynne B. Bacarro,

F.P.A. Demeterio, III, Jonathan Vergara Geronimo, and Patricia Bettina L. Peliño http://www.kritike.org/journal/issue 19/angeles et al december2016.pdf ISSN 1908-7330

(cc) BY-NC-ND 
bilang kabuuan at hindi laging kabalangkas sa daynamiks ng mga heterosekswal. Pinagtangkaan din ng pelikula na magbukas ng pagninilay ukol sa saradong pagtingin ng relihiyon sa mga praktika ng homosekswalidad at humihingi ng pagkilalang bahagi sila ng sambayanang Kristiyano. Mahihinuha rin na hindi mahalaga ang kategorisasyon, leybeling at pagpapangalan sa paghubog ng malayang identidad ng gender. Ang pagpapakahulugan sa gender ay nakabatay sa politikal at malay na pagpapasyang paulit-ulit na isinasagawa at inaangkin sa pagkalesbiyan, hinuhubog sa patuloy na paglaban sa ipinapataw na kaayusan hanggang sa maitanghal ang lesbiyan na hindi bukod o kaiba, kundi isang karaniwang indak at pamumukadkad na mahalagang tugon at hamon sa tunay na malayang lipunan.

Department of Filipino, De La Salle University, Philippines Department of Filipino, Univerity of Santo Tomas, Philippines Department of Filipino, Central Luzon State University, Philippines Department of Philosophy, De La Salle University, Philippines

\section{References}

Alfonso, Grac Javier, "Rome and Juliet: No Stereotyping of Women who Love Women," in Manunuri ng Pelkiulang Pilipino (30 October 2014), $<$ http://www.manunuri.com/reviews/rome and juliet no stereotyp ing of women who love women>, 12 March 2016.

Butler, Judith, Gender Trouble: Feminism and the Subversion of Identity (New York: Routledge, 1990).

Cantor, Olivia, “Lesbiana, Lesbiana, Paano Ka Isinasapelikula: Ilang Pagmumuni sa Pagsasadula ng mga Lesbiana sa Pelikulang Filipino," in Tabi-tabi sa Pagsasantabi: Kritikal na Tala ng mga Lesbiana at Bakla sa Sining, Kultura, at Wika, ed. by Eugene Y. Evasco, Roselle V. Pineda, and Rommel B. Rodriguez (Quezon City: University of the Philippines Press, 2003).

Guieb, Eulalio III, "Mga Anyo ng Sineng Filipino: Paghahanay at Paglilinaw ng mga Kinagisnan at mga Tunguhin," in Sining ng Sineng Filipino (Quezon City: UP Sentro ng Wikang Filipino, 2009).

Lumbera, Bienvenido, "Ang Pelikula sa Lipunang Filipino, Ang Lipunang Filipino sa Pelikula," in Writing the Nation/Pag-akda ng Bansa (Quezon City: University of the Philippines Press, 2010).

Oslon, Gary A. and Lynn Worsham, "Changing the Subject: Judith Butler's Politics of Radical Resignification," in Politics of Possibility:

(c) 2016 Judith R. Angeles, Tracie Kathlynne B. Bacarro, F.P.A. Demeterio, III, Jonathan Vergara Geronimo, and Patricia Bettina L. Peliño http://www.kritike.org/journal/issue 19/angeles et al december2016.pdf 


\section{PERFORMATIVITY AT PAGKALESBIYAN}

Encountering the Radical Imagination (New York: Paradigm Publishers, 2007).

Pangilinan, Sharon Anne, "Ang Pagdaloy sa Kasaysayan at Kasaysayan ng Pagdaloy ng Panitikang Lesbiyana ng Pilipinas," in Likhaan: The Journal of Contemporary Philippine Literature, 3 (2009).

Tolentino, Rolando B., "Lalaking Pin-up, GRO, at Macho Dancer" In Lalaking Pin-up, GRO, at Macho Dancer: Ang Pagtatanghal ng Pagkalalaki sa Edad ng Globalisasyon (Pasig City: Anvil Publishing, Inc., 2004).

(C) 2016 Judith R. Angeles, Tracie Kathlynne B. Bacarro,

F.P.A. Demeterio, III, Jonathan Vergara Geronimo, and Patricia Bettina L. Peliño http://www.kritike.org/journal/issue 19/angeles et al december2016.pdf ISSN 1908-7330

\section{(cc) EY-NC-ND}

